\title{
Orientation of vortices in a superconducting thin film: Quantitative comparison of spin-polarized neutron reflectivity and magnetization
}

\author{
S.-W. Han, ${ }^{1, *}$ J. Farmer, ${ }^{2}$ H. Kaiser, ${ }^{2}$ P. F. Miceli, ${ }^{1, \dagger}$ I. V. Roshchin, ${ }^{3}$ and L. H. Greene ${ }^{3}$ \\ ${ }^{1}$ Department of Physics and Astronomy, University of Missouri-Columbia, Columbia, Missouri 65211 \\ ${ }^{2}$ Missouri University Research Reactor, Columbia, Missouri 65211 \\ ${ }^{3}$ Department of Physics, University of Illinois at Urbana-Champaign, Urbana, Illinois 61801
}

(Received 25 February 2000)

\begin{abstract}
We present a quantitative comparison of the magnetization measured by spin-polarized neutron reflectivity (SPNR) and dc magnetometry on a $1370 \AA$ A-thick Nb superconducting film. As a function of magnetic field applied in the film plane, SPNR exhibits reversible behavior whereas the dc magnetization shows substantial hysteresis. The difference between these measurements is attributed to a rotation of the vortex magnetic field out of the film plane as the applied field is reduced. SPNR measures only the magnetization parallel to the film plane, whereas dc magnetization is strongly influenced by the perpendicular component of magnetization when there is a slight sample tilt; thus combining the two techniques allows one to distinguish two components of magnetization in a thin film.
\end{abstract}

\section{INTRODUCTION}

The current transport properties of type-II superconductors depend critically on the mobility of vortices and, therefore, the subject of vortex pinning has attracted considerable attention. ${ }^{1}$ Since the addition or removal of a vortex requires an encounter with the superconductor's surface, the interaction of vortices with surfaces and interfaces play an important role in the current transport properties. ${ }^{2,3}$ For thin-film superconductors, the surface has a substantial effect on vortex behavior. In this case, a large anisotropy between vortices parallel or perpendicular to the film plane might be anticipated. For example, recent studies of artificially layered superconducting thin films suggest that vortices parallel to the surface can order spatially due to the interaction with the surface ${ }^{4}$.

In studies of thin-film superconductors it is useful to apply the magnetic field parallel to the surface. This geometry can, however, be problematic for experiments. A magnetization measurement presents special difficulties due to the large demagnetization factor perpendicular to the surface combined with a small sample tilt angle that is unavoidable. On the other hand, local probe techniques, such as the Bitter method, scanning probe techniques, and Lorentz microscopy, are constrained to measure the magnetic flux as it emerges through the surface (i.e., vortices are perpendicular to the surface). Alternatively, small-angle neutron-diffraction methods require large volume samples so that thin films cannot be studied. Since the selection rules for the specular reflection of spin-polarized neutrons from a thin film guarantees that only the magnetization parallel to the surface is measured, spin-polarized neutron reflectivity (SPNR) should be useful for measuring the magnetization of thin-film superconductors.

The application of SPNR to thin-film superconductors was demonstrated by Felcher et al. ${ }^{5}$ where the London penetration length $\lambda_{L}$ was measured for $\mathrm{Nb}$. Further refinement of this technique for $\mathrm{Nb}$ by Zhang et $a .^{6}{ }^{6}$ included the effect of the electron mean free path on $\lambda_{L}$. The same technique was also used to determine $\lambda_{L}$ for high-temperature superconducting oxides. ${ }^{7}$ All of these studies were performed at low field, below $H_{c 1}$. Recently, it was demonstrated ${ }^{8,9}$ that SPNR is also useful at higher fields, above $H_{c 1}$, where it can yield information on vortices. In particular, Han et al. ${ }^{8}$ extracted the average vortex density for the field parallel to a thin film of $\mathrm{YBa}_{2} \mathrm{Cu}_{3} \mathrm{O}_{7-\mathrm{x}}$ (YBCO) and a large hysteresis loop was observed.

In this paper, we compare SPNR and dc magnetization measurements on a $\mathrm{Nb}$ thin film for the field applied parallel to the surface. The two measurements exhibit different field dependences: hysteresis is observed for the dc magnetization, whereas the SPNR is reversible. The difference is attributed to the magnetic field of vortices that is generated perpendicular to the film plane as the applied field is decreased. By quantitatively comparing the SPNR and magnetization measurements, we have deduced the rotation of the magnetic field as a function of the applied field.

\section{EXPERIMENTAL DETAILS}

A Nb/Al thin-film bilayer was grown by sputter deposition on a $1-\mathrm{cm}^{2}(1 \overline{1} 02)$ single-crystal $\mathrm{Al}_{2} \mathrm{O}_{3}$ substrate. ${ }^{10} \mathrm{~A}$ 50-h pumpdown in a UHV-compatible stainless-steel vacuum chamber was preformed with simultaneous substrate baking at $520^{\circ} \mathrm{C}$. Before deposition a cold trap was filled with liquid nitrogen, providing a base pressure of less than $1 \times 10^{-8}$ Torr. Sputter deposition was performed under a 7 $\times 10^{-3}$-Torr atmosphere of Ar. With the substrate temperature held at $470{ }^{\circ} \mathrm{C}$, a 1370 - $\AA$-thick layer of $\mathrm{Nb}$ was deposited at a rate of $8 \AA / \mathrm{sec}$. Without breaking vacuum, the substrate temperature was then reduced to $120^{\circ} \mathrm{C}$, and a $760-\AA$-thick layer of $\mathrm{Al}$ was deposited in situ on the $\mathrm{Nb}$ surface. The superconducting transition temperature was de- 


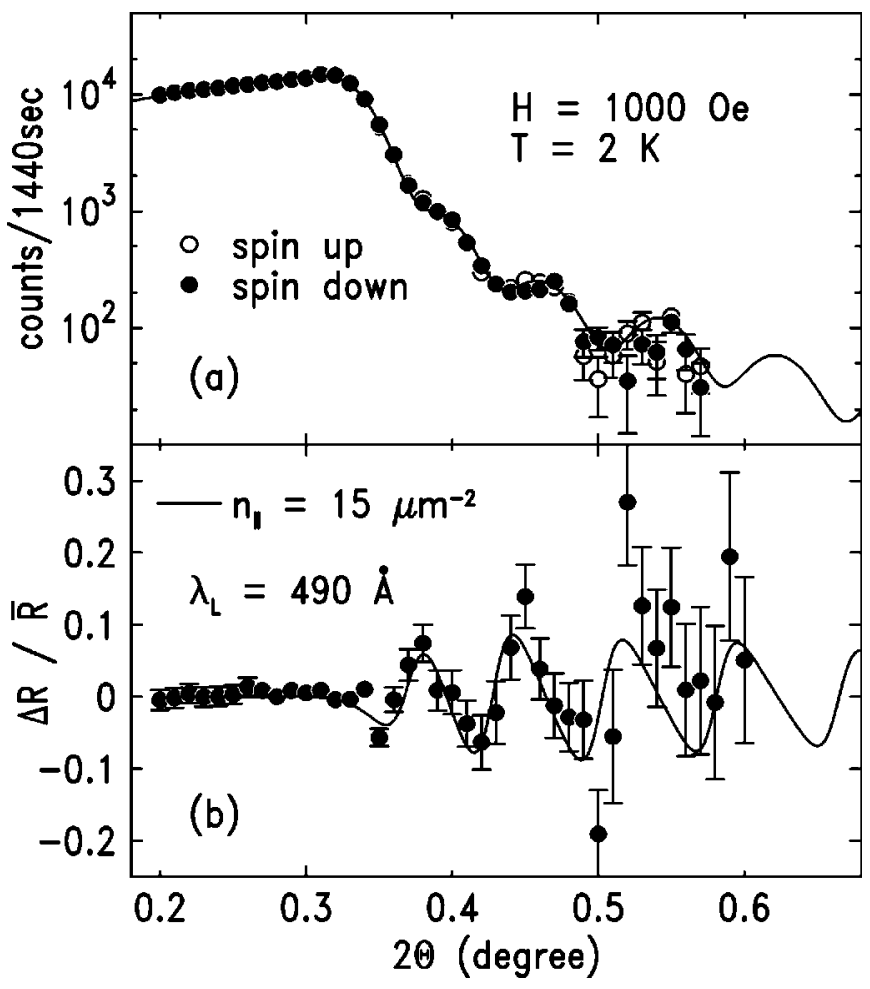

FIG. 1. (a) The number of counts are measured as a function of scattering angle for spin-up and -down neutrons reflected from a $\mathrm{Al} / \mathrm{Nb} / \mathrm{Al}_{2} \mathrm{O}_{3}$ film at $2 \mathrm{~K}$ and $1000 \mathrm{Oe}$. (b) The difference between the spin-up and -down reflectivity divided by the average reflectivity for the two spin states, $\Delta R / \bar{R}$, is obtained from the data in (a). The solid curves are calculated using the model of Ref. 8 , with a magnetic screening length of $490 \AA$ and a uniform vortex density of $15 \mu \mathrm{m}^{-2}$.

termined to be $T_{c}=9.25 \pm 0.25 \mathrm{~K}$ from the magnetization measured at $50 \mathrm{Oe}$, which is consistent with $T_{c}=9.11 \mathrm{~K}$ (transition width $0.06 \mathrm{~K}$ ) found by four-probe resistivity measured on an adjacently grown sample. The residual resistivity ratio was measured to be $\rho_{300 \mathrm{~K}} / \rho_{10 \mathrm{~K}}=25.4$.

The SPNR measurements were performed using the GANS reflectometer ${ }^{11}$ at the Missouri University Research Reactor (MURR) with a neutron wavelength of $2.35 \AA$. A supermirror was employed to select the spin state of the neutron beam to be perpendicular to the scattering plane, and the spin state could be switched using a flipper coil located downstream from the mirror. A polarization analyzer was not used. The beam width at the sample position, $\simeq 0.23 \mathrm{~mm}$, and the angular divergence of the beam, $0.018^{\circ}$, were measured in the scattering plane. During the data analysis, the instrumental resolution as well as the incident beam polarization efficiency, 93\%, were taken into account. The specular reflectivity was consecutively measured for spin-up and -down neutrons near the critical angle for total external reflection. The sample was mounted in a cryostat having a superconducting split-coil magnet where the field was applied perpendicular to the scattering plane and parallel to the sample surface. The sample was always cooled to $2 \pm 0.2 \mathrm{~K}$ in zero magnetic field before a field was applied.

A Quantum Design superconducting quantum interference device (SQUID) magnetometer was used for the magnetization measurements which were performed on the same

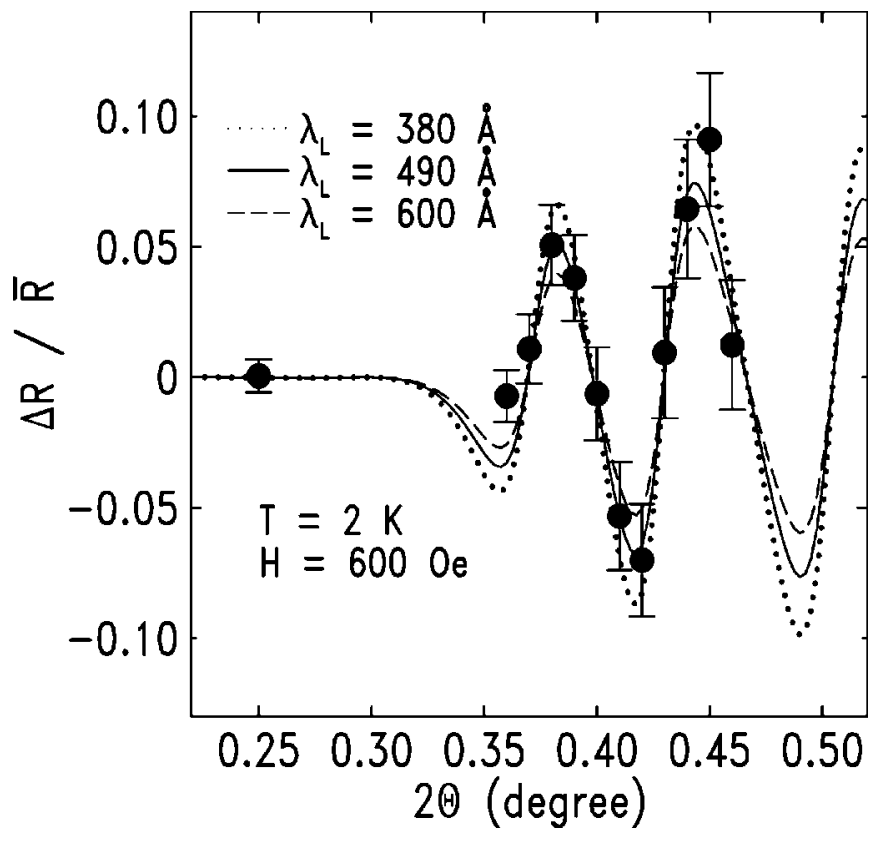

FIG. 2. The London penetration length was determined to be $\lambda_{L}=490 \pm 110 \AA$ by measuring $\Delta R / \bar{R}$ as a function of angle at 600 Oe and $2 \mathrm{~K}$, where the vortex density is negligible. The curves are calculated for different screening lengths without including vortices.

sample used for the neutron experiments. This sample was cut to $0.36 \times 0.26 \mathrm{~cm}^{2}$, and placed in the magnetometer cryostat such that the sample surface was near parallel to the applied field. The tilt angle between the sample surface, and the applied field was set when it was mounted in the magnetometer. The angular reproducibility was $\pm 0.25^{\circ}$ and the zero angle was determined from a fit to the angular dependence of the dc susceptibility measured at low field. The reported magnetization curves were obtained for the sample cooled in zero magnetic field.

\section{RESULTS}

The number of specularly reflected spin-up and down neutrons, measured as a function of the scattering angle $2 \theta$ for the sample held at $2 \mathrm{~K}$ and $1000 \mathrm{Oe}$, is shown in Fig. 1(a). The interference fringes arise from the finite thickness of the film. A least-squares fit using a reflectivity model ${ }^{12}$ yields the $\mathrm{Nb}$ film thickness $(1370 \pm 10 \AA)$ as well as the $\mathrm{Al} / \mathrm{Nb}(30 \pm 10 \AA)$, and $\mathrm{Nb} / \mathrm{Al}_{2} \mathrm{O}_{3}(2 \pm 1 \AA)$ interface roughnesses. Since the neutron-scattering cross section of $\mathrm{Al}$ is small relative to $\mathrm{Nb}$ and the neutron absorption in $\mathrm{Al}$ is negligible, the Al layer had little effect on the measurement. $\mathrm{X}$-ray specular reflectivity, measured on the same sample, gave an Al thickness of $760 \pm 30 \AA$ and a roughness of 70 $\pm 16 \AA$ at the air/Al interface, and the other quantities were consistent with the neutron measurement. The magnetic contribution to the neutron reflectivity can be observed by plotting the difference between the spin-up and -down reflectivities, $\Delta R$, divided by the average of the spin-up and -down reflectivity, $\bar{R}$, as shown in Fig. 1(b).

The London penetration length $\lambda_{L}$ for this sample was obtained from a SPNR measurement at small applied field (600 Oe), as shown in Fig. 2. Since the contribution of vor- 


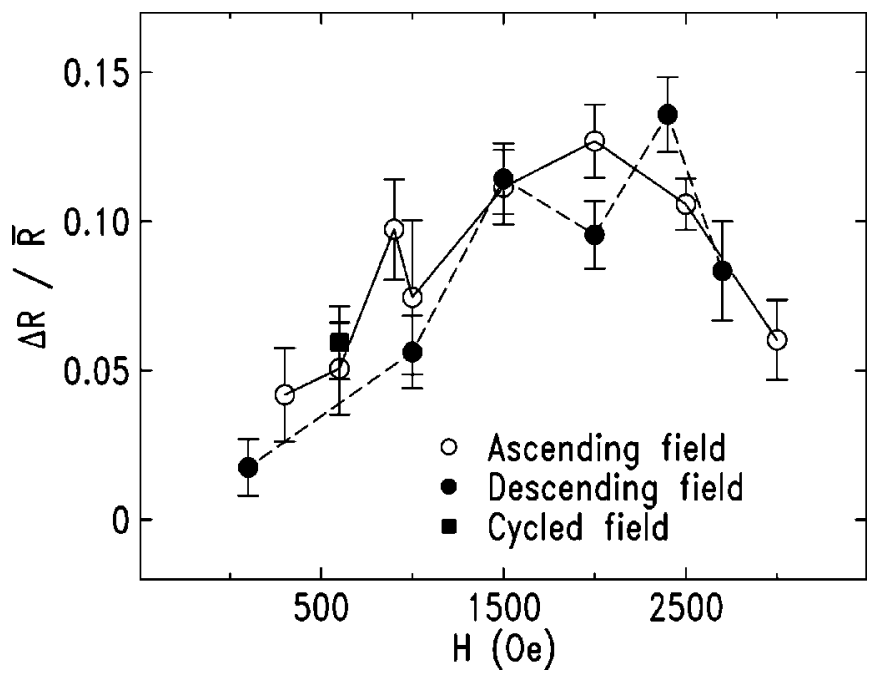

FIG. 3. The field dependence of $\Delta R / \bar{R}$ was measured at a single angle $2 \theta=0.38^{\circ}$, for ascending and descending field at $2 \mathrm{~K}$. The lines are a guide to the eye. London screening dominates at low field, but above $\sim 1000$ Oe the introduction of vortices leads to saturation and the eventual reduction of $\Delta R / \bar{R}$. No hysteresis is observed upon reducing the field. The data point given by the solid square was obtained after cycling the applied field from 0 to 3000 Oe, to $-3000 \mathrm{Oe}$, and finally to $600 \mathrm{Oe}$, which was the field during the measurement.

tices to the magnetization is negligible at this field, the data were $\mathrm{fit}^{8}$ assuming no vortices. This yields $\lambda_{L}=490$ $\pm 110 \AA$, in agreement with previous work. ${ }^{5,6}$

In order to observe the effect of vortices, the reflectivity was measured as a function of applied field at a single position of $2 \theta=0.38^{\circ}$, where $\Delta R / \bar{R}$ exhibits the first maximum. Figure 3 shows the results for ascending and descending fields. With ascending field (open circles), it is seen that

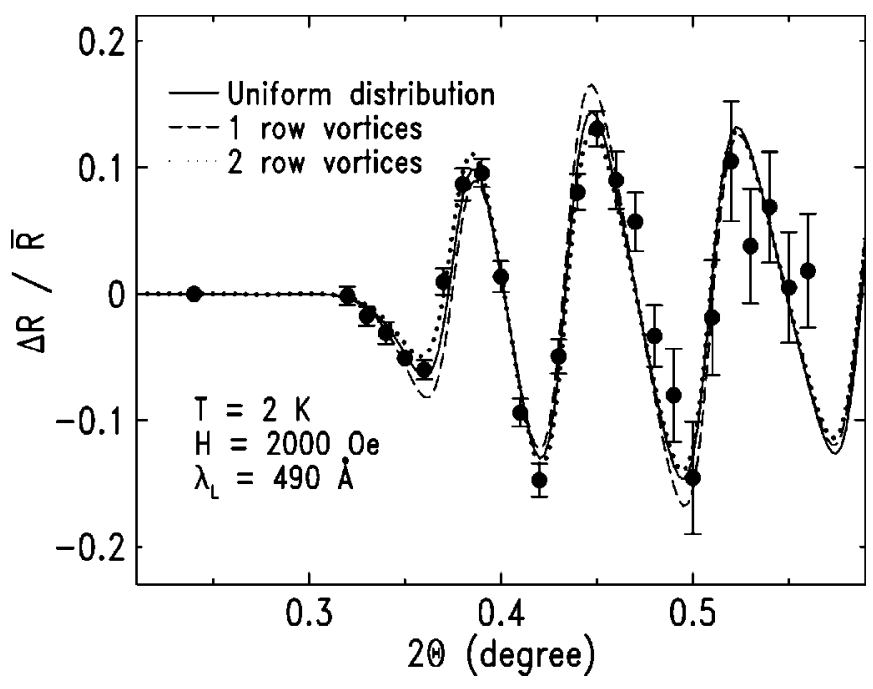

FIG. 4. $\Delta R / \bar{R}$ is measured as a function of angle at 2000 Oe and $2 \mathrm{~K}$ after reducing the field from 3000 Oe. It is shown that the analysis is independent of the particular choice of vortex distribution. The curves are best fits for different spatial distributions of vortices: uniform distribution with $n_{\|}=40 \mu \mathrm{m}^{-2}$, vortices in a single row at the center of the film with $n_{\|}=28 \mu \mathrm{m}^{-2}$, and vortices in a double row about the center of the film with $n_{\|}=33 \mu \mathrm{m}^{-2}$.

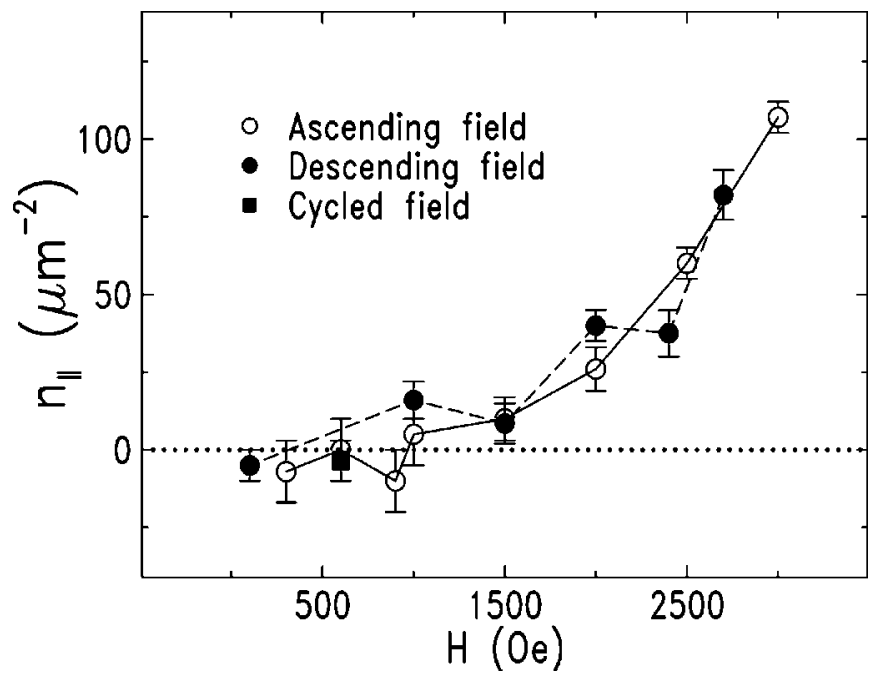

FIG. 5. The vortex density $n_{\|}$as a function of applied field is determined at $2 \mathrm{~K}$ from the SPNR data in Fig. 3 assuming a uniform spatial distribution of vortices, $\lambda_{L}=490 \AA$, and the model of Ref. 8 . These results show that $n_{\|}$is independent of whether the applied field is ascending or descending. The data point given by the solid square was obtained after cycling the applied field from 0 to 3000 Oe to -3000 Oe to 600 Oe.

$\Delta R / \bar{R}$ increases steadily until $\sim 1500$ Oe, where it begins to decrease as large numbers of vortices enter the sample. It can also be seen that the data for descending field follow the ascending field data and there is no remanence at low field. This reversible behavior is quite different from the previous SPNR study of a YBCO film, where $\Delta R / \bar{R}$ exhibited a large hysteresis loop. ${ }^{8}$

The data were analyzed to extract the vortex density using

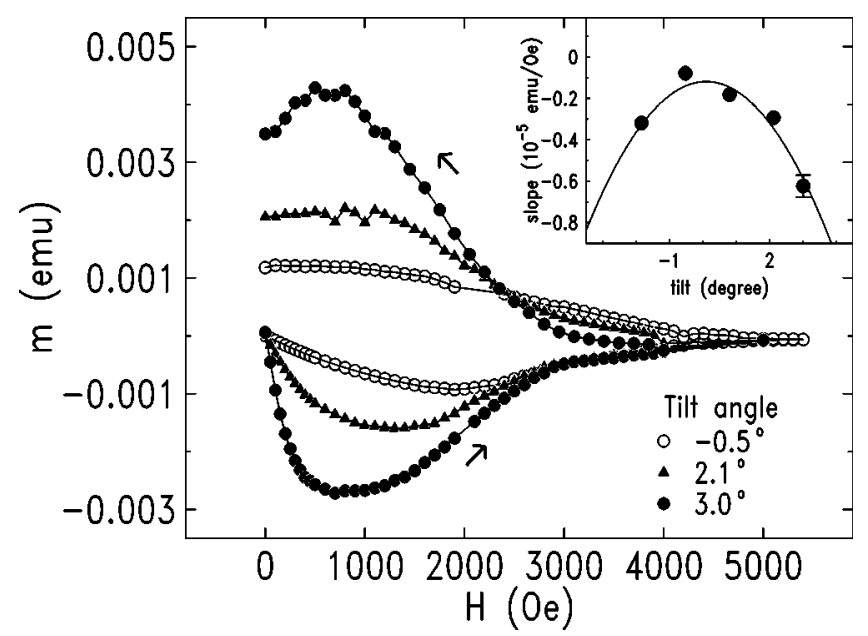

FIG. 6. The magnetic moment determined by the SQUID magnetometer and measured as a function of applied field at $2 \mathrm{~K}$ exhibits hysteresis. The arrows indicate the order in which the data were collected, starting from an initially zero-field-cooled sample. These results depend on the angle of tilt between the applied field direction and the film plane. The inset shows the initial slope (linear region without vortices at small field) of the magnetic moment as a function of the tilt angle. Demagnetization effects quantitatively explain the low-field angle dependence and the solid curve is a best fit to Eq. (3.5). 
a model presented previously. ${ }^{8}$ Although this requires the spatial distribution of the vortices as input information, the analysis of the present experiments does not depend on the choice of distribution, as shown in Fig. 4 for data taken at 2000 Oe after the sample had been subjected to a field of 3000 Oe. The three fitted curves correspond to different spatial distributions: a uniform distribution (solid curve) with a vortex density of $40 \mu \mathrm{m}^{-2}$, vortices arranged in a single row in the middle of the film (dashed curve) with a density of $28 \mu \mathrm{m}^{-2}$, and vortices arranged in a double row (dotted curve) with a density of $33 \mu \mathrm{m}^{-2}$. The given densities were chosen to yield a best fit for each spatial distribution. There is only a small difference between the curves, because the interference between the reflections from the front and back interfaces of the superconducting film dominates over the contribution from the vortex spatial distribution. This effect depends on the relative nuclear scattering lengths of the layers, and was not observed previously for $\mathrm{YBCO} / \mathrm{SrTiO}_{3}$.

Using the magnetic screening length $\lambda_{L}=490 \AA$ and a uniform distribution of vortices in the model of Ref. 8 , the average vortex density parallel to the surface, $n_{\|}$, was determined from the data of Fig. 3. The result, shown in Fig. 5, gives $H_{c 1} \sim 1000 \mathrm{Oe}$, and the vortex density is found to increase steadily at higher field. Also, $n_{\|}$exhibits reversible behavior. The full angle-dependent curves for $\Delta R / \bar{R}$ are calculated and compared with the data in Figs. 1(b), 2, and 4-the good agreement demonstrates that it is sufficient to measure $\Delta R / \bar{R}$ at a single angle in order to obtain $n_{\|}$.

We now discuss magnetization measurements that provide additional information on the vortex behavior. The SQUID magnetization measured as a function of applied field for different tilt angles is shown in Fig. 6. For each angle, the sample was first zero field cooled to $2 \mathrm{~K}$. The magnetization was subsequently measured from zero to the maximum field, and then to zero field. Above $H_{c 1}$ the magnetization curves do not show the maxima that have been observed in some artificially layered superconductors, ${ }^{4}$ sug- gesting there are no transitions of the vortex distribution in the film. These data, as well as other measurements performed at higher field (not shown), permit an estimate of the upper critical field $H_{c 2} \sim 5300 \pm 300$ Oe. From this, the coherence length can be calculated: $\xi=\sqrt{\Phi_{o} /\left(2 \pi H_{c 2}\right)}=250$ $\pm 8 \AA$. This is somewhat smaller than the $390 \AA$ obtained for $\mathrm{Nb}$ by Pronin et al., who also reported the London penetration depth to be $350 \AA \AA^{13}$ Since the samples were grown in the same sputtering system using the same procedure, the difference might arise from the fact that the two techniques determine the coherence length in somewhat different ways. Using the magnetic screening length $(490 \AA)$ and coherence length $(250 \AA)$, we estimate the lower critical field in the thin-film limit, ${ }^{14} \quad H_{c 1}=\Phi_{o} K_{0}\left(\xi / \lambda_{L}\right) /\left(4 \pi \lambda_{L}^{2}\right) \times(1$ $\left.+2 e^{-t / 2 \lambda_{L}}\right)$, to be $\sim 1000 \mathrm{Oe}$, which agrees well with both the SPNR (Fig. 5) and the magnetization measurements (from the smallest tilt angle of $-0.5^{\circ}$ in Fig. 6).

The most striking features of the SQUID data are the large hysteresis, in sharp contrast to the SPNR measurement, as well as the strong dependence on tilt angle. Both features are interrelated, and we first discuss the effect of angle.

If a magnetic field $H_{\perp}$ is applied perpendicular to the surface of a superconductor, then the magnetization perpendicular to the surface $M_{\perp}$ is strongly affected by the geometry according to ${ }^{14,15}$

$$
4 \pi M_{\perp}=-\frac{H_{\perp}-n_{\perp} \Phi_{o}}{(1-N)},
$$

where $N$ is the demagnetization factor, $n_{\perp}$ is the component of vortex density oriented perpendicular to the surface, and $\Phi_{o}$ is the flux quantum: $h c / 2 e=20.679 \mathrm{G} \mu \mathrm{m}^{2}$. Note that $n_{\perp} \Phi_{o}$ is just the component of magnetic field perpendicular to the film plane. For a field applied parallel to the surface of a superconductor, assuming a uniform distribution of vortices, the magnetization parallel to the surface $M_{\|}$is obtained by direct integration of the equations in Ref. 8 ,

$$
\begin{aligned}
4 \pi M_{\|}= & \frac{1}{t} \int_{-t / 2}^{t / 2}\left[H_{\|}\left\{\frac{\cosh \left(z / \lambda_{L}\right)}{\cosh \left(t / 2 \lambda_{L}\right)}-1\right\}+\frac{n_{\|} \Phi_{o}}{2 \lambda_{L}} \int_{-t / 2}^{t / 2}\left\{e^{-\left|z-z^{\prime}\right| / \lambda_{L}-e^{(z-t / 2) / \lambda_{L}} \frac{\sinh \left(\left[z^{\prime}+t / 2\right] / \lambda_{L}\right)}{\sinh \left(t / \lambda_{L}\right)}}\right.\right. \\
& \left.\left.+e^{-(z+t / 2) / \lambda_{L}} \frac{\sinh \left(\left[z^{\prime}-t / 2\right] / \lambda_{L}\right)}{\sinh \left(t / \lambda_{L}\right)}\right\} d z^{\prime}\right] d z=-\left(H_{\|}-n_{\|} \Phi_{o}\right)\left\{1-\frac{2 \lambda_{L}}{t} \tanh \left(\frac{t}{2 \lambda_{L}}\right)\right\},
\end{aligned}
$$

where $n_{\|}$is the component of vortex density parallel to the surface. The magnetic field generated parallel to the surface is $n_{\|} \Phi_{e f f}$, where

$$
\Phi_{e f f}=\Phi_{o}\left\{1-\frac{2 \lambda_{L}}{t} \tanh \left(\frac{t}{2 \lambda_{L}}\right)\right\}
$$

is the average effective flux quantum of a vortex that is screened by image fields due to the surfaces. For this sample,
$\Phi_{\text {eff }}=0.37 \Phi_{o}$. Figure 7(a) shows the magnetization obtained from the SPNR results of Fig. 5 using Eq. (3.2).

In the SQUID magnetometer the magnetization is measured along the direction of the applied field, so when the sample surface is slightly tilted from the applied field by an angle $\theta$ a projection of the components of magnetization parallel and perpendicular to the surface will be measured:
$M_{\|} \cos \theta+M_{\perp} \sin \theta$. 

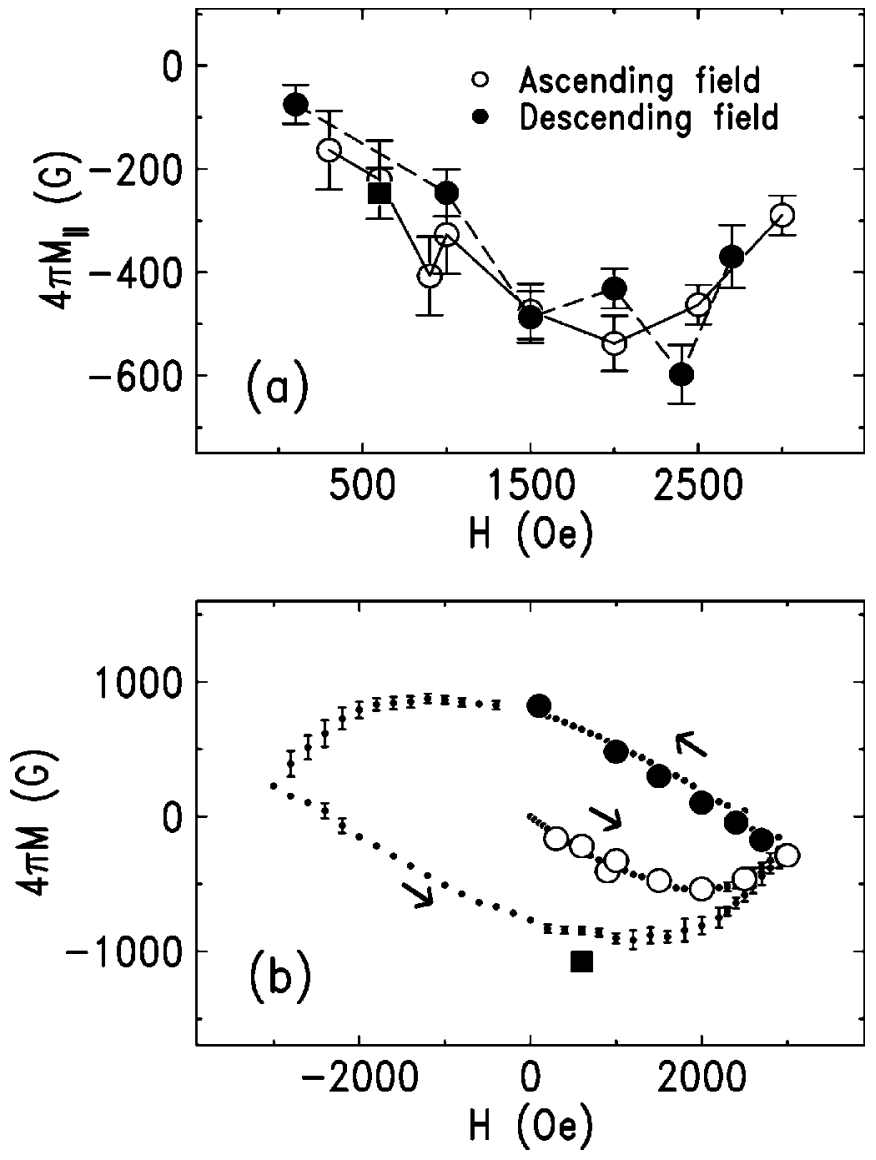

FIG. 7. (a) The magnetization as a function of applied field is determined from the SPNR data in Fig. 5 using Eq. (3.2). (b) Quantitative agreement between the SQUID and SPNR measurments is achieved using the model described in the text. In (b) the small data points are the SQUID magnetization measured at $2 \mathrm{~K}$ as a function of applied field using the same field history as the SPNR experiment. The arrows indicate the order in which the data were collected. The open circles are the magnetization from the ascending field SPNR data replotted from (a), showing that the SPNR and SQUID measurements lead to the same result: for ascending field, the vortices are parallel to the film plane. The large solid circles are the descending field SPNR data converted to the conditions of the SQUID measurement assuming the model described in the text. The solid square comes from the SPNR data point measured after cycling the field to -3000 Oe.

This is demonstrated quantitatively for our data in the low-field Meissner regime, below $H_{c 1}$. In this regime, the magnetization of a superconducting film at an angle with the applied field will be

$$
4 \pi M_{o}=-H \cos ^{2} \theta\left\{1-\frac{2 \lambda_{L}}{t} \tanh \left(\frac{t}{2 \lambda_{L}}\right)\right\}-\frac{H \sin ^{2} \theta}{1-N} .
$$

The inset to Fig. 6 shows the slope of the low-field magnetic moment data of Fig. 6 as a function of tilt angle, where the solid curve is the result of a least-squares fit to Eq. (3.5) with two free parameters $N$ and a scale factor. The latter permits an accurate determination of the magnetization, which also contains small instrumental effects. ${ }^{16}$ As can be seen, the curve represents the data quite well and we obtain $N$
$=0.998 \pm 0.0015$, which is slightly larger than the value 0.994 estimated for our sample geometry. ${ }^{17}$

More generally, we can include the effect of vortices on the magnetization measured with the SQUID by substituting Eqs. (3.1) and (3.2) into Eq. (3.4), which gives

$$
4 \pi M=4 \pi M_{o}+n_{\|} \Phi_{e f f} \cos \theta+\frac{n_{\perp} \Phi_{o} \sin \theta}{1-N}
$$

where $M_{o}$ is the Meissner term in the absence of vortices, given by Eq. (3.5).

The conclusion is that the magnetometer is highly sensitive to the component of magnetization perpendicular to the film. Although this component diminishes with decreasing $\theta$, the demagnetization factor causes the denominator of Eq. (3.1) to be small. Thus the SQUID measurements can, even for very small angles of tilt, be dominated by the magnetization perpendicular to the film. This effect is also much larger for the vortex contribution than in the Meissner regime, since the Meissner effect diminishes as $\sin ^{2} \theta$ but the vortex contribution diminishes more slowly, as $\sin \theta$. Therefore, we conclude that the irreversibility observed in the SQUID measurement is due to $M_{\perp}$, since, the SPNR shows $M_{\|}$to be reversible.

In order to quantitatively compare the SPNR and SQUID experiments, the SQUID magnetization was measured by following the same field history as the SPNR measurement, as shown by the small data points in Fig. 7(b). This was done for the sample tilt angle nominally set to zero. The open circles in Fig. 7(b) are the ascending field SPNR data replotted from Fig. 7(a). As can be seen, the SPNR and SQUID measurements give quantitatively the same magnetization for the virgin ascending field, indicating that the magnetization and vortices are parallel to the film plane. Moreover, the quantitative agreement between the two data sets also indicates that sample tilt effects as well as any contribution from vortices perpendicular to the surface are insignificant for the virgin ascending field.

The SQUID and SPNR measurements differ only subsequent to the virgin magnetization, which suggests that a magnetic field due to vortices is generated out of the film plane as the applied field is reduced. Thus, for a descending field, the data were analyzed using two components of magnetic field, one parallel $\left(n_{\|} \Phi_{e f f}\right)$ and one perpendicular $\left(n_{\perp} \Phi_{o}\right)$ to the film plane. The parallel magnetic field is obtained directly from the SPNR measurement, and it is desireable to obtain the perpendicular field from the SQUID data. However, as can be seen from Eq. (3.6), the perpendicular field contribution to the SQUID magnetization also depends on the sample tilt angle, which is not precisely known, so that an additional piece of information is required. It is found that good quantitative agreement between the SQUID and SPNR data can be obtained by assuming that the sum of the parallel and perpendicular magnetic fields is equal to the maximum trapped field, $n_{\| \max } \Phi_{\text {eff }}=n_{\|} \Phi_{\text {eff }}+n_{\perp} \Phi_{o}$, where $n_{\| \max }$ is the maximum vortex density parallel to the film plane, which occurs at the maximum applied field. The large solid data points in Fig. 7(b) show the SQUID magnetization predicted from the SPNR data using this relationship and a tilt angle of $0.12^{\circ}$. Note that this angle is essentially zero to within the experimental error of setting the angle; however, 


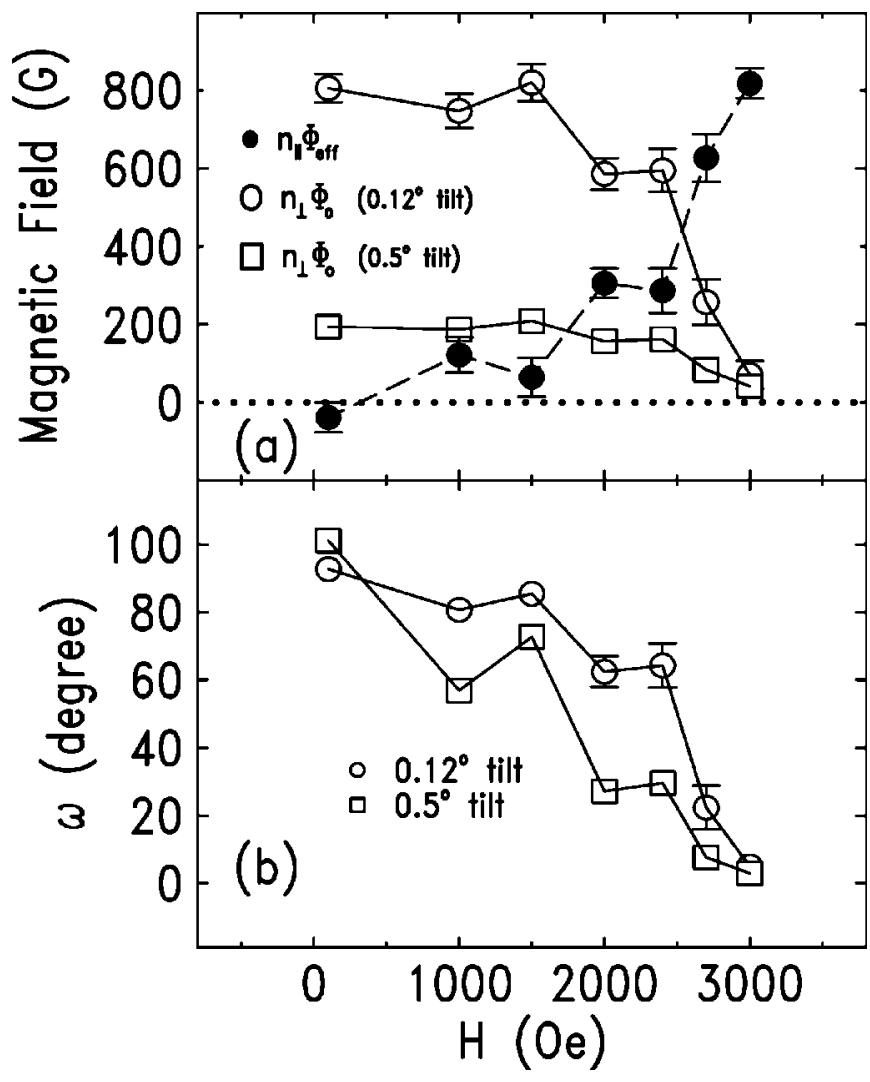

FIG. 8. For descending applied field, the SQUID and SPNR measurements are used to determine the magnetic field in the sample. (a) The magnetic field parallel to the surface, given by the solid circles, is obtained directly from the SPNR measurement. The perpendicular magnetic field, shown by the open circles $\left(0.12^{\circ}\right)$ and open squares $\left(0.5^{\circ}\right)$, is obtained by solving Eq. (3.6) using the SQUID and SPNR data and by assuming the given sample tilt angles. (b) shows the angle between the magnetic field and the surface for the two assumed angles of sample tilt.

its finite size qualitatively impacts the shape of the magnetization curve by determining the size of the hysteresis loop. The solid square SPNR data point was measured at $600 \mathrm{Oe}$ after cycling the field to -3000 Oe. In that case, the maximum density of vortices was assumed to be the same as was measured by SPNR at $3000 \mathrm{Oe}$, and this also gives good agreement with the SQUID data. An alternative model where the total vectorial magnetic field is conserved, corresponding to $\left(n_{\| \max } \Phi_{e f f}\right)^{2}=\left(n_{\|} \Phi_{e f f}\right)^{2}+\left(n_{\perp} \Phi_{o}\right)^{2}$, gave a substantially poorer comparison between the SPNR and SQUID data, although the qualitative trend was similar.

The perpendicular magnetic field can also be obtained independent of such models if a specific tilt angle is assumed. In this case, we have chosen two angles: $0.5^{\circ}$ and $0.12^{\circ}$. The former is twice the maximum that could have been set experimentally, and the latter is the value obtained from the above model (which we take as the lower limit because a smaller angle would unphysically give $n_{\perp \max } \Phi_{o}$ $\left.>n_{\| \max } \Phi_{e f f}\right)$. The result of this analysis is given in Fig. 8(a) which shows the individual magnetic-field components. Figure 8(b) shows the resulting angle between the magnetic field and the film plane: $\omega=\tan ^{-1}\left(n_{\perp} \Phi_{o} / n_{\|} \Phi_{e f f}\right)$.

\section{DISCUSSION AND CONCLUSIONS}

The good quantitative agreement between the SQUID and SPNR data using the above analysis supports the basic conclusion that the initial magnetic field of the vortices is parallel to the film plane and that it subsequently rotates perpendicular to the film plane as the applied field is removed. Although the analysis presented in Fig. 7(b) assumes that the trapped magnetic field is strictly conserved, one cannot draw this conclusion because of the uncertainty in the sample tilt angle. Figure 8 demonstrates the range of perpendicular magnetic fields that can be obtained, which depend upon the assumed sample tilt. Assuming the maximum tilt leads to a lower limit on the maximum trapped perpendicular magnetic field: $n_{\perp \max } \Phi_{o}=0.25 n_{\| \max } \Phi_{\text {eff }}$. In this limiting scenario, $75 \%$ of the magnetic field leaves the sample when the applied field is reduced to zero. In any case, it is safe to conclude that a significant fraction of the magnetic field is trapped at remanance, and that it is oriented perpendicular to the film plane.

Based on previous studies, these results are different from what one might expect. For example, our SPNR investigation $^{8}$ of a YBCO thin film showed a large hysteresis loop and remanence, indicating a strong preference for the vortices to remain parallel to the film plane. From the smaller coherence length of $\mathrm{YBCO}$ one expects significantly stronger vortex pinning in $\mathrm{YBCO}$ than $\mathrm{Nb}$. However, the rotation of the trapped magnetic field in $\mathrm{Nb}$ rather than the removal of vortices is not expected. Other studies of anisotropic superconductors ${ }^{18}$ have found that the vortex density perpendicular to the surface is essentially proportional to the projection of the applied field. However, those studies were performed on thick crystals, and the vortices were examined upon field cooling. Given the isotropy of $\mathrm{Nb}$, we expect that the observed magnetic field rotation is due to the thin-film nature of the sample (thickness $/ \lambda_{L} \sim 2.8$ ).

We can speculate on two surface-related mechanisms that give a lower equilibrium energy for vortices oriented perpendicular to the film plane. First the vortex density in the perpendicular direction is no more than $37 \%$ of the maximum parallel vortex density (because $n_{\perp \max } \Phi_{o} \leqslant n_{\| \max } \Phi_{\text {eff }}$ ) and this leads to a lower vortex-vortex repulsion for vortices reoriented perpendicular to the film plane. A second mechanism relates to the weaker vortex binding energy (one-body interaction) for the parallel geometry where there are two energy contributions arising from the surface: ${ }^{19}$ a repulsive term due to the vortex interaction with the surface-screening field, and an attractive term due to the interaction with the image vortex. The repulsive term is proportional to $H$, and will dominate at higher field, thereby leading to smaller binding energy for vortices parallel to the surface. Whether these mechanisms are relevant will depend on kinetics as well as crystalline anisotropy. It could be that the latter plays a role in the YBCO thin film. ${ }^{8}$

Also of interest are the pinning, rotation, and nucleation behavior of vortices in this geometry. One might expect, to a first approximation, that the mechanism for vortex rotation is similar to vortex removal: the vortex line must move toward a surface. However, the vortices appear to rotate rather than exit the sample, suggesting that opposite ends of a vortex line penetrate the opposite planar surfaces of the film. Once 
rotated, the vortex pinning is much stronger in the perpendicular geometry. ${ }^{3}$ Vortex rotation alone, however, does not explain these results. Given the magnitude of the perpendicular magnetic field found in Fig. 8, many additional vortices must be generated when the field is reduced because the planar area of the film is nearly $10^{5}$ times larger than the area of the film's edge. It might be that, with decreasing applied field, the segment of a vortex that is parallel to the surface decreases in length (effectively giving a rotation, since, the "threading" segment perpendicular to the surface is constant in length) while, simultaneously, new vortices are nucleated in order to increase the number of vortex threads that penetrate the planar surface. Clearly, further theoretical and ex- perimental work is necessary in order to gain a better understanding of the vortex behavior in this geometry.

\section{ACKNOWLEDGMENTS}

Support is gratefully acknowledged (P.F.M., S.W.H., J.F.) from the Midwest Superconductivity Consortium (MISCON) under DOE Grant No. DE-FG02-90ER45427, the NSF DMR 96-23827, and (L.H.G., I.V.R.) from DOE Grant No. DE FG02 96 ER45439. We are also grateful to John Clem for helpful discussions. The SPNR experiments were performed at the Missouri University Research Reactor (MURR).
*Present address: Department of Physics, University of Washington, Seattle, Washington 98195.

'Author to whom correspondence should be addressed.

${ }^{1}$ G. Blatter, M. V. Feigel'man, V. B. Geshkenbein, A. I. Larkin, and V. M. Vinokur, Rev. Mod. Phys. 38, 1125 (1994).

${ }^{2}$ Y. Paltiel, D. T. Fuchs, E. Zeldov, Y. N. Myasoedov, H. Shtrikman, M. L. Rappaport, and E. Y. Andrei, Phys. Rev. B 58, 14763 (1998); Ernst Helmut Brandt, ibid. 58, 6506 (1998); R. G. Mints, I. B. Snapiro, and E. H. Brandt, ibid. 55, 8466 (1997); David. A Huse, ibid. 46, 8621 (1992).

${ }^{3}$ E. Zeldov, J. R. Clem, M. McElfresh, and M. Darwin, Phys. Rev. B 49, 9802 (1994).

${ }^{4}$ J. Guimpel, L. Civale, F. de la Cruz, J. M. Murduck, and I. K. Schuller, Phys. Rev. B 38, 2342 (1988); S. H. Brongersma, E. Verweij, N. J. Koeman, D. G. de Groot, R. Griessen, and B. I. Ivlev, Phys. Rev. Lett. 71, 2319 (1993); P. Lobotka, I. Vávra, R. Senderák, D. Machajdík, M. Jergel, S. Gaži, E. Rosseel, M. Baert, Y. Bruynseraede, M. Forsthuber, and G. Hilscher, Physica C 299, 231 (1994); S. M. Yusuf, E. E. Fullerton, R. M. Osgood II, and G. P. Felcher, J. Appl. Phys. 83, 6801 (1998); S.-W. Han, J. Farmer, P. F. Miceli, G. P. Felcher, R. Goyette, G. Kiehne, and J. Ketterson (unpublished).

${ }^{5}$ G. P. Felcher, R. T. Kampwirth, K. E. Gray, and R. Felici, Phys. Rev. Lett. 52, 1539 (1984).

${ }^{6}$ H. Zhang, J. W. Lynn, C. F. Majkrzak, S. K. Satija, J. H. Kang, and X. D. Wu, Phys. Rev. B 52, 10395 (1995).

${ }^{7}$ A. Mansour, R. O. Hilleke, G. P. Felcher, R. B. Lainbowitz, P. Chaudhari, and S. S. P. Parkin, Physica B 156\&157, 867 (1989); S. V. Gaponov, E. B. Dokukin, D. A. Korneev, E. B. Klyuenkov, W. Löbner, V. V. Pasyuk, A. V. Petrenko, Kh. Rzhany, and L. P. Chernenko, Pis'ma Zh. Éksp. Teor. Fiz. 49, 277 (1989) [JETP Lett. 49, 316 (1989)]; V. Lauter-Pasyuk, H. J. Aksenov, E. L. Kornilov, A. V. Petrenko, and P. Leiderer, Physica B 248, 166 (1998).

${ }^{8}$ S.-W. Han, J. F. Ankner, H. Kaiser, P. F. Miceli, E. Paraoanu, and L. H. Greene, Phys. Rev. B 59, 14692 (1999).

${ }^{9}$ V. Lauter-Pasyuk, H. J. Lauter, M. Lorenz, V. L. Aksenov, and P.
Leiderer, Physica B 267-268, 149 (1999).

${ }^{10}$ L. H. Greene, A. C. Abyeta, I. V. Roshchin, I. K. Robinson, J. F. Dorsten, T. A. Tanzer and P. W. Bohn, in Spectroscopic Studies of Superconductors, edited by I. Bozovic and D. van der Marel, SPIE Proceedings No. 2696 (SPIE, Bellingham, WA, 1996), p. 215; L. H. Greene, J. F. Dorsten, I. V. Roshchin, A. C. Abeyta, T. A. Tanzer, G. Kuchler, W. L. Feldmann, and P. W. Bohn, Czech. J. Phys. 46, 3115 (1996); I. V. Roshchin, Ph.D. thesis, University of Illinois, 2000.

${ }^{11}$ H. Kaiser, K. Hamacher, R. Kulasekere, W.-T. Lee, J. F. Ankner, B. DeFacio, P. Miceli, and D. L. Worcester, in Inverse Optics III, SPIE Proceedings No. 2241 (SPIE, Bellingham, WA, 1994), pp. 78-89.

${ }^{12}$ T. P. Russell, Mater. Sci. Rep. 5, 171 (1990); P. F. Miceli, in Semiconductor Interfaces, Microstructures and Devices: Properties and Applications, edited by Z. C. Feng (Institute of Physics, Bristol, 1993), p. 87.

${ }^{13}$ A. V. Pronin, M. Dressel, A. Pimenov, A. Loidl, I. V. Roshchin, and L. H. Greene, Phys. Rev. B 57, 14416 (1998).

${ }^{14}$ A. A. Abrikosov, Fundamentals of the Theory of Metals (NorthHolland, Amsterdam, 1988).

${ }^{15}$ A. P. Malozemoff, L. Krusin-Elbaum, D. C. Cronemeyer, Y. Yeshurun, and F. Holtzberg, Phys. Rev. B 38, 6490 (1988).

${ }^{16}$ M. McElfresh, S. Li, and R. Sager, Effects of Magnetic Field Uniformity on the Measurement of Superconducting Sample (Quantum Design, 1996); M. McElfresh, Fundamentals of Magnetism and Magnetic Measurements (Quantum Design, 1994).

${ }^{17}$ D. Craik, Magnetism Principles and Applications (Wiley, New York, 1995), p. 298.

${ }^{18}$ H. F. Hess, C. A. Murray, and J. V. Waszczak, Phys. Rev. B 50, 16528 (1994); C. A. Bolle, P. L. Gammel, D. G. Grier, C. A. Murray, D. J. Bishop, D. B. Mitzi, and A. Kapitulnik, Phys. Rev. Lett. 66, 112 (1991).

${ }^{19}$ P. G. de Gennes, Superconductivity of Metals and Alloys (Addison-Wesley, New York, 1989); T. P. Orlando and K. A. Delin, Foundations of Applied Superconductivity (AddisonWesley, New York, 1991). 